\title{
Pulse Interval Modulation-based Method to Extract the Respiratory Rate from Oscillometric Cuff Pressure Waveform During Blood Pressure Measurement
}

\author{
Yihan Gui ${ }^{1}$, Fei Chen ${ }^{1}$, Alan Murray ${ }^{2}$, Dingchang Zheng ${ }^{3}$ \\ ${ }^{1}$ Department of Electrical and Electronic Engineering, \\ Southern University of Science and Technology, Shenzhen, China \\ ${ }^{2}$ School of Electrical and Electronic Engineering and Faculty of Medical Sciences, \\ Newcastle University, Newcastle upon Tyne, UK \\ ${ }^{3}$ Health and Wellbeing Academy, Anglia Ruskin University, Chelmsford, UK
}

\begin{abstract}
Respiratory frequency has been extensively used to assess health status. This study aimed to evaluate two methods of extracting the respiratory rate from oscillometric cuff pressure pulses (OscP) during blood pressure (BP) measurement, which was compared with reference respiration signal (Resp).

OscP and Resp were simultaneously recorded on 20 healthy subjects during the linear cuff deflation period of BP measurement. Reference Resp was obtained from a chest magnetometer and OscP from an electronic pressure sensor connected to the cuff. Two de-modulation methods were developed by using the peak or valley positions of the OscP waveform to measure pulse intervals, from which the respiration modulation signal was derived. Statistical analysis showed that, in comparison with the Resp, there was no significant difference (-0.001 Hzfor the peak-based method, and $0.001 \mathrm{~Hz}$ for valley-based method), and their corresponding limits of agreement were $-0.08 \mathrm{~Hz}$ to 0.08 $\mathrm{Hz}$ and $-0.10 \mathrm{~Hz}$ to $0.11 \mathrm{~Hz}$, respectively. There was also a high correlation between Resp and respiratory frequencies extracted from OscP waveform, with the correlation coefficients of 0.7 for both methods.

In conclusion, the present work demonstrated that, during BP measurement, respiratory frequency can be accurately derived from using either peak or valley point to characterize pulse intervals.
\end{abstract}

\section{Introduction}

It has been well known from published studies that respiration is one of the major factors affecting blood pressure (BP) measurements [1, 2]. Even though the underlying mechanism of how respiration affects BP measurement has not been fully understood, it is generally accepted that respiration influences the central venous pressure through the chest expansion and compression, and then affects stroke volume [3].

Previously published work has showed the possibility of extracting respiratory frequency from the electrocardiography (ECG) signal [e.g., 4-6]. Since the automatic BP measurement devices are extensively used in healthcare, BP measurement is more easily accessible than ECG measurement both in hospital and at home.

Oscillometric cuff pressure pulses (OscP) are widely used in automated non-invasive BP measurement devices. Based on the cardiorespiratory phenomenon, Zheng et al. [3] has demonstrated the modulation effect of respiration on OscP during standard oscillometric BP measurement. This has inspired several follow up studies to extract respiratory frequency from OscP, based on oscillometric pulse interval changes during the cuff deflation of BP measurement [e.g., 7]. This study aimed to develop two more pulse interval-based methods from OscP to derive respiratory frequency during $\mathrm{BP}$ measurement, and test its accuracy in comparison with that from reference respiration signals (Resp).

\section{Method}

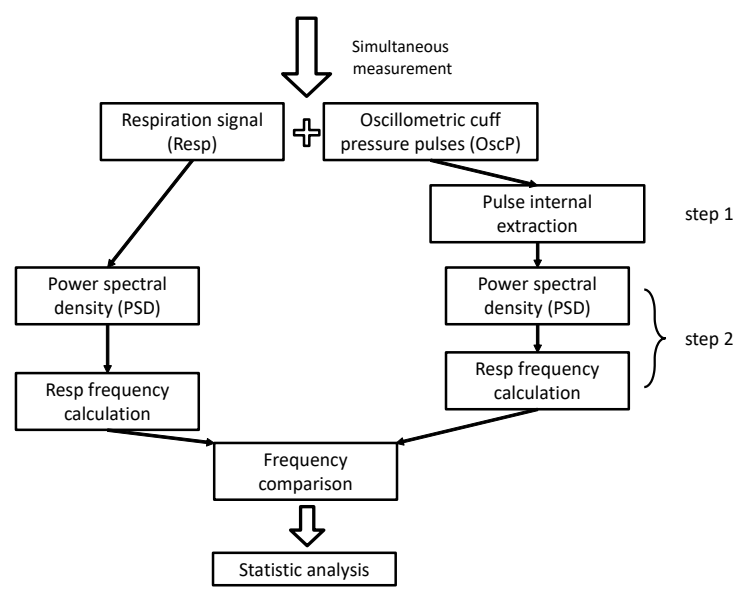

Figure 1. Procedure of the experiment in this work. 


\subsection{Experiment overview}

As shown in figure 1, multi-physiological parameters were simultaneously recorded (including Resp and OscP) from subjects, which were processed separately. Next, the respiration frequencies from Resp and OscP were statistically analyzed and compared to evaluate the efficiency of the methods developed in this work.

\subsection{Subjects}

$\mathrm{BP}$ and respiration measurements were performed on 20 healthy subjects (10 males and 10 females; aged from 23 to 65 years). Their mean \pm SD of age, height and weight were $39 \pm 16$ years, $175 \pm 8 \mathrm{~cm}$ and $72 \pm 10 \mathrm{~kg}$, respectively. This study was carried out according to the Declaration of Helsinki (1989) of the World Medical Association, and was approved by Newcastle and North Tyneside NHS Research Ethics Committee. Informed and written consent was obtained from all subjects. Anonymized data was analysed.

\subsection{Experimental setup}

For each subject, OscP and Resp were simultaneously recorded during the linear cuff deflation period (from 200 to $30 \mathrm{mmHg}$ ) of $\mathrm{BP}$ measurement. Reference Resp (assumed as ground truth) was measured by a chest magnetometer for detecting chest wall movement and OscP by an electronic pressure sensor connected to the cuff. Manual systolic and diastolic blood pressures (SBP and DBP) were measured by a trained operator, which was used to define the boundary of the modulation signal. Six repeated measurements were performed for each participant. A one-minute break time was given between every two consecutive measurements to stabilize cardiovascular parameters.

\subsection{Signal processing}

Signal processing for extracting respiratory frequencies from OscP involves two steps: (1) Extract the pulse interval modulation signals from OscP by the two demodulation methods (i.e., peak-based and valley-based methods), as exemplified in Figs. 2 and 3; and. (2) Calculate the respiratory frequency from the power spectral density (PSD) of the corresponding pulse interval modulation signal, as shown in Fig. 4. Peak-based method and valley-based method had the identical procedure in step 2. The details about the implementation of each signal processing step are described below.

Step 1. Extracting pulse interval modulation signals from OscP by peak-based and valley-based methods.

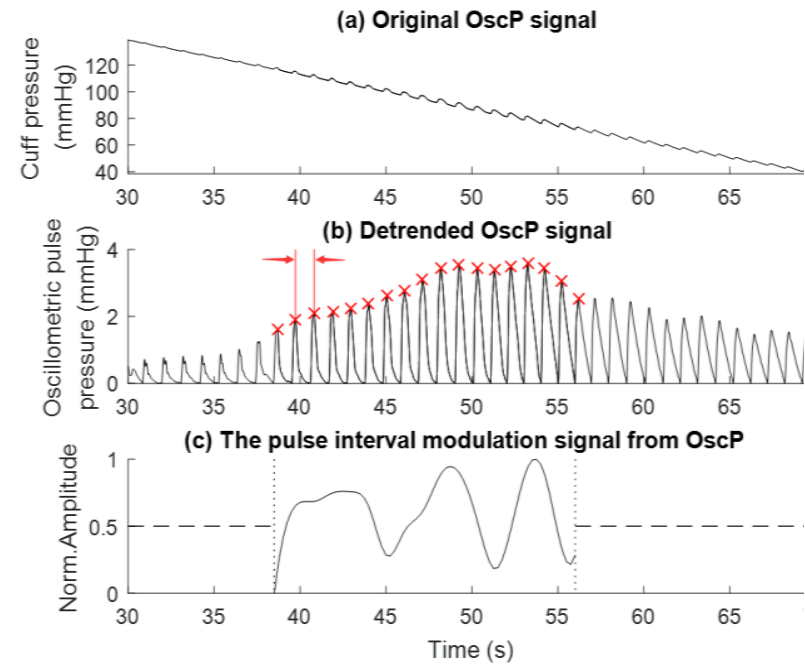

Figure 2. Peak-based method of extracting the pulse interval modulation signal from OscP. (a) Original OscP signal between 30s and 70s. (b) De-trended OscP signal. Each red $\mathrm{x}$-mark indicates the maximum amplitude point (peak) of each OscP pulse during the time period between SBP and DBP. The red arrows indicate a pulse interval. (c) Normalized pulse interval modulation signal (the solid line) extracted from the data of OscP during the period between SBP and DBP. Dashed lines: no signal. Dotted lines: time boundary of the modulation signal.

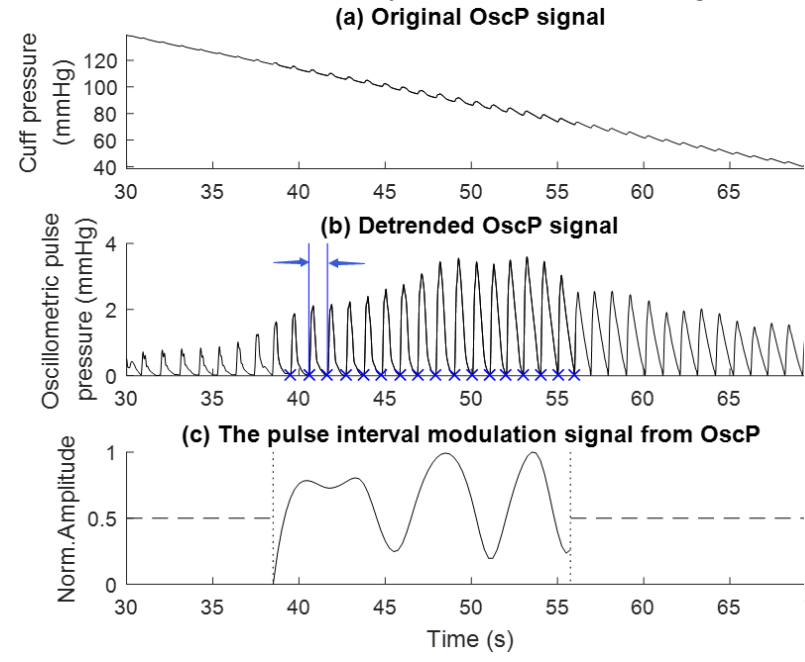

Figure 3. Valley-based method of extracting the pulse interval modulation signal from OscP. (a) Original OscP signal. (b) De-trended OscP signal. Each blue x-mark indicates the minimum amplitude point (valley) of each OscP pulse. The blue arrows indicate a pulse interval. (c) Normalized pulse interval modulation signal.

Figure 2 and Figure 3 illustrate the procedure of extracting pulse interval modulation signal from OscP signal using peak-based and valley-based methods separately. Before the de-modulation procedure, OscP 
signals had been filtered by a low-pass filter $(0-10 \mathrm{~Hz})$ which was designed for high frequency noise removal. As shown in Fig. 2 (a), the deflating baseline cuff pressure is superimposed by small cuff pressure oscillations that arise from beat-to-beat BP pulses from the arteries under the cuff. The deflating baseline cuff pressure was removed through de-trending processing. Figure 2 (b) and 3 (b) indicate the de-trended OscP signal. In this study, only the pulses during the period between SBP and DBP were then used for extracting respiratory frequency. Pulse intervals of the OscP signal were derived from the time interval of the adjacent maximum or minimum amplitude points of OscP. Finally, the pulse interval modulation signal from OscP was constructed by cubic spline interpolation with a sample rate of $4 \mathrm{~Hz}$ on the pulse interval series, as shown in Figure 2 (c) and 3(c).

\section{Step 2. Respiratory frequency calculation.}

The normalized PSDs of Resp and pulse interval modulation signals from OscP were estimated by Welch periodogram between 0.1 and $0.5 \mathrm{~Hz}$ with the frequency resolution of $0.001 \mathrm{~Hz}$. Respiratory frequency from Resp $\left(f_{R}\right)$ and $\operatorname{OscP}\left(f_{P}\right.$ and $\left.f_{V}\right)$ were estimated as the frequency at the peak of the PSD. As shown in Fig. 4, the peak of each PSD is marked with a star and the frequency corresponding to the maximum amplitude point was recognized as the respiratory frequency. In this example, the respiratory frequencies extracted from $\mathrm{OscP}$ are closed to the reference respiratory frequency with the difference of $0.008 \mathrm{~Hz}$ in Fig. 4 (a) and $0.003 \mathrm{~Hz}$ in (b).
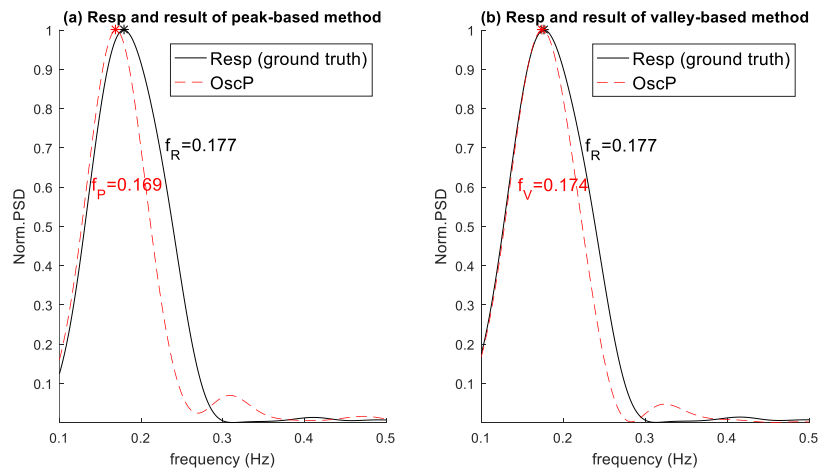

Figure 4. Normalized PSDs of Resp (the solid lines) and pulse interval modulation signals (the dashes lines) from OscP by using (a) peak-based method and (b) valley-based method. Red or black star of each plot indicates the peak of the corresponding PSD. $\mathrm{f}_{\mathrm{R}}$ : respiratory frequency from ground truth, $\mathrm{f}_{\mathrm{P}}$ and $\mathrm{f}_{\mathrm{v}}$ : respiratory frequency from OscP by the peakbased or valley-based method.

\subsection{Statistical analysis}

Statistical analysis with paired t test between the derived and reference respiratory frequencies was performed, with Bland-Altman plots produced. The correlation coefficients were used to assess the correlation between $\mathrm{f}_{\mathrm{R}}$ and $\mathrm{f}_{\mathrm{P}}$ or $\mathrm{f}_{\mathrm{V}}$. Limit of Agreement (LOA) was also used, which was defined as average difference \pm 1.96 times of standard deviation of difference, i.e., $[\mu-1.96 \sigma, \mu+1.96 \sigma],(\mu$ : average difference, $\sigma$ : standard deviation).

\section{Results}
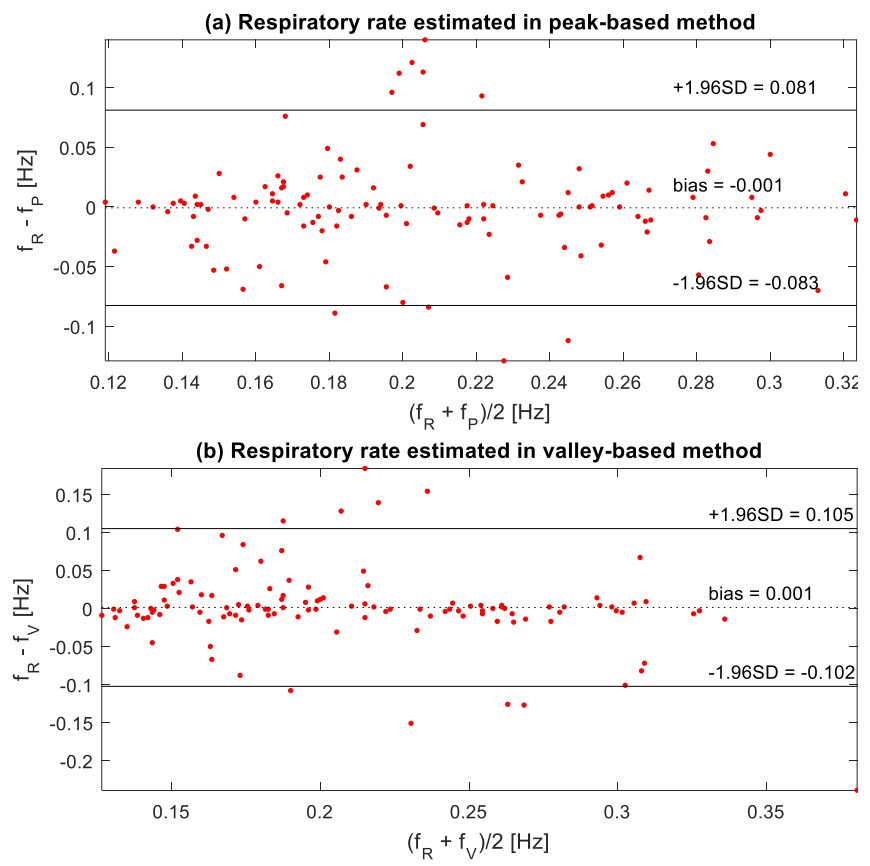

Figure 5. Bland-Altman difference plots of respiratory frequency estimation from OscP by using (a) peak-based method and (b) valley-based method. Dotted black lines indicate bias and solid black lines indicate 1.96-SD limits of agreement.

The results showed that there was no statistically significant difference (all $\mathrm{p}>0.05$ ) for the respiratory frequencies derived in this study in comparison with reference respiratory frequency. Table 1 lists its mean bias, mean absolute bias and LOA for evaluating the accuracy of estimating respiratory frequency with $\mathrm{f}_{\mathrm{P}}$ and $\mathrm{f}_{\mathrm{V}}$, as well as their correlation coefficient. The mean biases were near

Table 1. Comparison of respiratory frequency from peak- and valley-based methods with reference respiratory frequency

\begin{tabular}{ccccc}
\hline & $\begin{array}{c}\text { Correlation } \\
\text { coefficient }\end{array}$ & $\begin{array}{c}\text { Mean } \\
\text { bias } \\
{[\mathrm{Hz}]}\end{array}$ & $\begin{array}{c}\text { Mean } \\
\text { absolute } \\
\text { bias [Hz] }\end{array}$ & LOA [Hz] \\
\hline $\mathrm{f}_{\mathrm{P}}$ & 0.69 & -0.001 & 0.027 & {$[-0.08,0.08]$} \\
\hline $\mathrm{f}_{\mathrm{V}}$ & 0.65 & 0.001 & 0.029 & {$[-0.10,0.11]$} \\
\hline
\end{tabular}

$* f_{\mathrm{P}}$ and $\mathrm{f}_{\mathrm{V}}$ : respiratory frequencies from OscP by the peak- and valley-based methods, respectively. 
zero and mean absolute biases were less than $0.03 \mathrm{~Hz}$, confirming the accuracy of extracting respiratory frequency from OscP based on both two methods. The correlation coefficients between $f_{R}$ and $f_{P}$ or $f_{V}$ were 0.69 and 0.65 separately, indicating a high correlation between the methods developed here with the reference technique.

Bland-Altman plots in Fig. 5 present respiratory frequency estimation errors of $f_{P}$ and $f_{V}$ in comparison with $\mathrm{f}_{\mathrm{R}}$ (ground truth) during BP measurement. Most recordings $\left(84 \%\right.$ of $f_{P}$ and $83 \%$ of $f_{V}$ ) agree with $f_{R}$ within $0.05 \mathrm{~Hz}$, indicating that it is reliable to extract respiratory frequency from OscP during BP measurement.

\section{Discussion and conclusion}

The present work developed and evaluated two pulse interval-based methods, for extracting respiratory frequency from oscillometric cuff pressure during the BP measurement. The results demonstrated that respiratory frequency can be accurately derived from using either peak or valley point to characterize pulse intervals.

This study extended and confirmed our previous work that respiratory frequency can be effectively extracted from oscillometric cuff pressure waveform and Korotkoff sounds during BP measurement [7]. Besides, our results showed that the proposed two magnitude-based methods in this study accurately estimated respiratory frequency during BP measurement, providing a solution for monitoring additional clinical parameter during BP measurement.

\section{Acknowledgements}

This study was supported by the National Natural Science Foundation of China (Grant No. 61571213) and by the Engineering and Physical Sciences Research Council (EPSRC) grants (reference number EP/I027270/1 and EP/F012764/1).

\section{References}

[1] Novak V, Novak P, De Champlain J, Le Blanc AR, Martin R, Nadeau R. Influence of respiration on heart rate and blood pressure fluctuations. J Appl Physiol 1993;74:617-626.

[2] Jagomagi K, Raamat R, Talts J, Lansimies E, Jurvelin J. Effect of deep breathing test on finger blood pressure. Blood Press Monit 2003;8:211-214.

[3] Zheng D, Di Marco LY, Murray A. Effect of respiration on Korotkoff sounds and oscillometric cuff pressure pulses during blood pressure measurement. Med Biol Eng Comput 2014;52:467-473.

[4] Mason CL, Tarassenko L. Quantitative assessment of respiratory derivation algorithms. International Conference of the IEEE Engineering in Medicine \& Biology Society, 2001;2:1998-2001.

[5] Schäfer A, Kratky KW. Estimation of breathing rate from respiratory sinus arrhythmia: Comparison of various methods. Ann Biomed Eng. 2008;36:476-485.

[6] Langley P, Bowers EJ, Murray A. Principal component analysis as a tool for analyzing beat-to-beat changes in ECG features: Application to ECG-derived respiration. IEEE Trans Biomed Eng 2010;57:821-829.

[7] Chen D, Chen F, Murray A, Zheng D. A method for extracting respiratory frequency during blood pressure measurement, from oscillometric cuff pressure pulses and Korotkoff sounds recorded during the measurement. International Conference of the IEEE Engineering in Medicine \& Biology Society 2016; 4268-4271.

Address for correspondence:

Prof Fei Chen

Department of Electrical and Electronic Engineering Southern University of Science and Technology Xueyuan Road 1088\#, Xili, Nanshan District, Shenzhen, China Email: fchen@sustc.edu.cn 